\title{
ORGANIZATIONAL LEARNING: A THEORY OF ACTION PERSPECTIVE
}

Ch. Argyris y D. A. Schön

«... Intervenir es entrar en un conjunto de relaciones en desarrollo con el propósito de ser útil. El tipo de ayuda en el que nos vamos a centrar consiste en aumentar la capacidad para una buena dialéctica organizativa - es decir, la capacidad de indagación organizativa para encajar errores, incongruencias e incompatibilidades en una teoría organizativa de la acción la cual necesariamente emerge a medida que el sistema organizativo/ambiental cambia» (p. 158).

«... Nuestra actividad en la intervención debe por tanto ocuparse de tres propósitos. Estos son: 1) ayudar al cliente a ser consciente y a descongelar sus teorías al uso del Modelo I y sistemas de aprendizaje O-I, y 2) educar al cliente a usar el Modelo II y a crear sistemas de aprendizaje O-II, para 3) usar este nuevo conocimiento con el fin de lograr una buena dialéctica organizativa.

Llamamos a la actividad de intervención que incluye las tres intenciones una intervención extensa» (p. 166).

\section{LOS COMPONENTES DEL PROCESO DE LA INTERVENCION EXTENSA}

Tal y como hemos visto, los clientes que mantienen teorías al uso del Modelo I y que están implicados en un sistema de aprendizaje organizativo 
O-I no suelen saber cómo desarrollar teorías al uso del Modelo II ni sistemas de aprendizaje O-II y, además, suelen desconocer su falta de habilidad para hacerlo. La actividad central en el proceso de intervención extensa es dominar el aprendizaje de bucle doble. En lugar de educar directamente a los clientes a descubrir-inventar-producir y generalizar soluciones de bucle doble, primero tenemos que ayudarles a ser conscientes de que son incapaces de hacerlo y de que no han sabido que eran incapaces. A continuación, debemos ayudarles a aprender cómo descubrir problemas de doble bucle que incluyen (tal y como hemos visto en el Capítulo 6) aprender a: descubrir cómo descubrir, inventar formas de descubrir, producir los inventos que llevan al descubrimiento y generalizar el aprendizaje creado como resultado de haber pasado por estos procesos. Después, el cliente debe pasar por fases similares de invención, producción y generalización.

Si el proceso de intervención tiene que ser extenso, los clientes tienen que aprender lo anterior no sólo para cambiar sus teorías particulares al uso, sino también para cambiar el sistema de aprendizaje O-I por el O-II. Con otras palabras, el aprendizaje de doble bucle debe producirse a nivel individual y a nivel sistémico. Esto último es desarrollado por los individuos que son los agentes del aprendizaje organizativo. Pero éstos son los mismos individuos que están intentando aprender a cambiar sus teorías al uso del Modelo I. Tal y como veremos, el aprendizaje oscila continuamente entre estos dos niveles. El aprendizaje puede ser iniciado en el nivel individual, pero necesariamente se mueve al nivel del sistema, después vuelve al individuo y de nuevo se empieza el ciclo.

Para que las personas puedan alejarse del Modelo I y del O-I, necesitan una idea de hacia dónde van (un modelo del estado final) y un modelo de cómo ir hacia él (el proceso de transformación). Por tanto, el segundo componente del proceso de intervención consiste en sacar a la superficie las inconsistencias entre el I y el II y O-I y O-II. El conflicto resultante produce la energía y la motivación para aprender. Los modelos pueden ser usados para informar a los individuos del grado de eficacia con que avanzan en dirección a los Modelos II y O-II y del grado en que están usando los procesos adecuados.

Primero, para aprender cómo aprender el doble bucle (a nivel individual o de sistema) los clientes deben ser conscientes de lo que ellos (y el sistema que representan) son incapaces de realizar. Esto significa que deben ser ayudados a descubrir su comportamiento y sus actividades actuales y las razones de por qué no conocen sus limitaciones actuales. Para lo cual, los miembros de la organización deben ser ayudados a descubrir su sistema de aprendizaje O-I. Para hacerlo posible tendrán que darse cuenta de sus teorías del Modelo I al uso, de los bucles inhibidores primarios y secundarios (a nivel grupal e intergrupal) y de las normas y reglas de la organización. Por tanto, el primer paso en un proceso de intervención extensa es un diagnóstico amplio de los problemas que tendrán que ser superados para crear un sistema O-II. De nuevo, si nuestro diagnóstico es correcto, es muy alta la probabilidad de que los clientes 
no sean capaces de realizar este diagnóstico por sí mismos. La información que necesitan tenderá a estar diseminada. Posiblemente existirá de forma vaga y no clara. Existirán muchas inconsistencias e incoherencias. Se requiere una gran habilidad para obtener información bajo estas circunstancias.

Pero la situación es incluso más complicada si nuestros clientes tratasen de llevar a cabo un diagnóstico extenso, lo harían equipados de teorías al uso que, en interacción con datos que ya son de antemano vagos, poco claros e inconsistentes, pueden hacer que los datos san aún más vagos, oscuros e inconsistentes. Es más, en su indagación estarán en gran parte inhibidos por los juegos de camuflaje y decepción que existen en su sistema O-I. El que los clientes lleven a cabo el diagnóstico es tanto como pedirles que violen las reglas y las normas de su sistema de aprendizaje. Si combinamos lo anterior con la inadvertencia del cliente de su incompetencia para diagnosticar sus teorías al uso o su sistema de aprendizaje, así como su falta de habilidad para inventar soluciones a los problemas descubiertos o para producir conclusiones novedosas, podemos predecir que nuestro proceso de intervención no será fácil para nuestro cliente.

En segundo lugar, los clientes no sabrán entonces cómo crear una buena dialéctica. Puesto que están programados con el Modelo I e inmersos en un Modelo O-I de sistema de aprendizaje, la probabilidad es alta de que tiendan a no saber cómo inventar un modelo cuyos supuestos básicos y valores guía cuestione los supuestos existentes y los valores dirigentes en la organización. Si supieran cómo hacer esto, ya tendrían la habilidad para el aprendizaje de bucle doble. De nuevo, los clientes tenderán a encontrarse en una situación que es difícil, frustrante y productora de tensión.

Por tanto, es necesario ayudar a los clientes a aprender a cómo manejar los sentimientos de sorpresa, inadecuación, incompetencia y dificultad. Estos sentimientos aparecerán cuando los clientes se den cuenta de lo disfuncionales que son sus teorías al uso, así como el sistema de aprendizaje que han creado y que continúan manteniendo. Además, necesitarán ayuda para aprender a manejar los sentimientos de indefensión y frustración que serán resultado de que, durante los primeros estadios de la intervención, casi cualquier acción que emprendan para cambiar sus teorías al uso del Modelo I o su sistema de aprendizaje O-I tenderá a ser contraproducente. Debido a su desconocimiento, no tenderán a darse cuenta de que esto es así hasta que ocurra.

Pero a medida que los clientes progresen, encontrarán nuevos dilemas. Cada nuevo descubrimiento parecerá destapar los tipos de problemas para los que no han sido educados o que han defendido que no existían en sus organizaciones, o que (si existían) parecían inabordables. Estos descubrimientos causan sentimientos añadidos de frustración y fracaso. Puesto que los clientes no saben ni cómo solucionar el problema sustantivo (ej., crear un sistema O-II) ni cómo manejar los sentimientos de fracaso y de frustración de forma eficaz, existe una gran probabilidad de que los hallazgos realizados puedan crear un proceso que culmine en fracaso o en la negación de que los problemas descubiertos son los que la organización debe trabajar. Por tanto, el proceso de 
intervención parece afirmar que los clientes deben ser expuestos a experiencias dolorosas para aprender. Otra condición, por tanto, es que los clientes aprendan a ver el dolor como una oportunidad para el desarrollo, y un cierto grado de miedo como un componente necesario del aprendizaje de bucle doble. Con otras palabras, los sentimientos que posiblemente han aprendido a lo largo de los años a considerar como malos y disfuncionales son los que ahora deben aprender a usar como plataforma para el aprendizaje y el cambio. Esto implica que los clientes estarán continuamente enfrentados a dilemas y que necesitarán aprender a usar estos dilemas como base para el aprendizaje.

A medida que se cumplan estas condiciones, el siguiente paso en el proceso de la intervención consiste en ayudar a los agentes a seleccionar unos pocos problemas instrumentales para los que puedan desarrollar soluciones. Las experiencias de éxito, obtenidas de estas innovaciones, pueden empezar a aumentar en el cliente el sentido de confianza en sí mismo, así como a disminuir la impresión de que los problemas son insuperables. Esto, a su vez, puede llevarles a desarrollar una perspectiva de tiempo y un nivel de aspiración más realista sobre el tiempo que les llevará aprender a la organización. Después se les ayuda a producir estas innovaciones en sus organizaciones y a seguir su desarrollo de forma que puedan corregir los errores que se produzcan, así como generalizar sobre acciones futuras.

A medida que los clientes empiezan a experimentar un éxito inicial en las condiciones de aprender a descubrir, se puede iniciar la siguiente fase, es decir, inventar soluciones a los problemas descubiertos. Para inventar de forma eficaz, los clientes tendrán que aprender a descubrir cómo inventar, cómo inventar soluciones, cómo producir estas soluciones y así en adelante. Esto implica, a su vez, que los clientes tenderán a experimentar ahora las mismas tensiones y frustraciones que experimentaron cuando aprendían a descubrir. Si han tenido un éxito, aunque sea pequeño, mientras aprendían a descubrir, entonces su confianza recién adquirida les ayudará a manejar los problemas de la invención de forma más eficaz. Es más, los mismos éxitos vividos en la fase anterior tenderán a reducir su sentido de inutilidad y de desesperanza porque ahora tienen la evidencia de que pueden superar y aprender de estas dificultades.

A medida que los clientes empiecen a ganar algún pequeño grado de confianza en su habilidad para descubrir e inventar tratarán de aprender a producir sus propias creaciones. De nuevo se encontrarán con las dificultades y tensiones antes descritas. Pero debido a sus éxitos anteriores probablemente se sentirán menos asustados por las dificultades y tensiones y esto aumentará la probabilidad de que lo hagan bien (Cap. 8, pp. 167-170). 


\section{CRITICA DE LIBROS}

